\title{
Quantitative Label-Free Imaging of Lipid Domains in Single Bilayers by Hyperspectral Coherent Raman Scattering
}

\author{
Alexander Nahmad-Rohen, David Regan, Francesco Masia, Craig McPhee, Iestyn Pope, \\ Wolfgang Langbein, and Paola Borri*
}

Cite This: Anal. Chem. 2020, 92, 14657-14666

Read Online

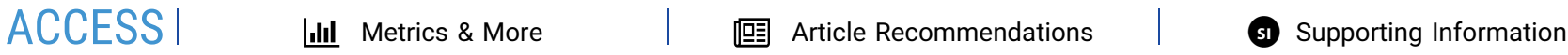

ABSTRACT: Lipid phase separation in cellular membranes is thought to play an important role in many biological functions. This has prompted the development of synthetic membranes to study lipid-lipid interactions in vitro, alongside optical microscopy techniques aimed at directly visualizing phase partitioning. In this context, there is a need to overcome the limitations of fluorescence microscopy, where added fluorophores can significantly perturb lipid packing. Raman-based optical imaging is a promising analytical tool for label-free chemically specific microscopy of lipid bilayers. In this work, we demonstrate the application of hyperspectral coherent Raman scattering microscopy combined
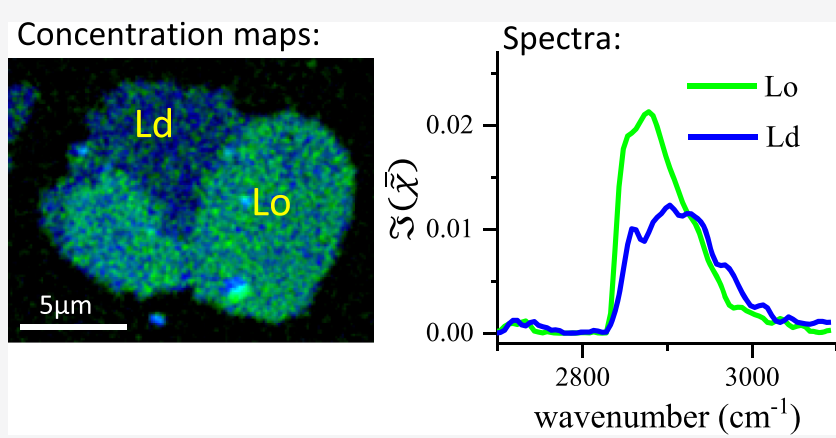
with a quantitative unsupervised data analysis methodology developed in-house to visualize lipid partitioning in single planar membrane bilayers exhibiting liquid-ordered and liquiddisordered domains. Two home-built instruments were utilized, featuring coherent anti-Stokes Raman scattering and stimulated Raman scattering modalities. Ternary mixtures of dioleoylphosphatidylcholine, sphingomyelin, and cholesterol were used to form phase-separated domains. We show that domains are consistently resolved, both chemically and spatially, in a completely label-free manner. Quantitative Raman susceptibility spectra of the domains are provided alongside their spatially resolved concentration maps.

\section{INTRODUCTION}

Lipid bilayers have been investigated for several decades to study lipid-lipid and lipid-protein interactions. ${ }^{1}$ They serve as model systems to aid our understanding of the heterogeneous organization of cellular membranes ${ }^{2}$ and in the investigation of many processes, including protein segregation in lipid domains, membrane protein function, drug-receptor interactions, and transmembrane transport. Notably, they are receiving increasing attention as building blocks in bottom-up synthetic biology approaches to creating artificial cells. ${ }^{3}$

A widely investigated physicochemical phenomenon of biomimetic membranes is the liquid-liquid phase coexistence occurring when saturated lipids and sterols condense to form a liquid-ordered $\left(\mathrm{L}_{\mathrm{o}}\right)$ phase which separates from a liquiddisordered $\left(\mathrm{L}_{\mathrm{d}}\right)$ phase rich in unsaturated lipids. ${ }^{4}$ This is an important phenomenon not only from a fundamental lipidbiophysics point of view but also in relation to the highly debated lipid raft hypothesis, which postulates that cholesterol (Chol)-rich ordered domains within the plasma membrane of cells serve as platforms for protein function and associated cell signaling and trafficking events. ${ }^{2}$ However, the quantitative characterization of these heterogeneous lipid domains in single bilayers is not trivial, and the presently utilized techniques have a number of drawbacks.
Atomic force microscopy (AFM) is a powerful tool to achieve nanometric topology resolution and has been utilized to distinguish the extremely small $(\sim 1 \mathrm{~nm})$ height differences between $L_{o}$ and $L_{d}$ lipid domains in bilayers. ${ }^{5,6}$ As a contactforce-based method, it must be utilized on supported bilayers adhering onto a substrate. Severe drawbacks of AFM are slow imaging speed and lack of chemical specificity.

Alternatively, optical phase-contrast techniques can provide information on the optical path length and, in turn, membrane thickness when combined with appropriate quantitative analysis. Especially, wide-field imaging techniques offer fast acquisition speeds and can interrogate supported bilayers ${ }^{7}$ as well as suspended bilayers such as giant unilamellar vesicles (GUVs). ${ }^{8}$ However, these methods are also not chemically sensitive.

Domain visualization in lipid membranes can be achieved using fluorescence microscopy with a range of fluorophore-

Received: July 27, 2020

Accepted: October 8, 2020

Published: October 22, 2020 
labelled lipids and can also feature spatial resolution below the diffraction limit $(<200 \mathrm{~nm})$ when combined with superresolution methods. The main limitations of this approach are photobleaching and perturbation to the native lipid behavior by the addition of the fluorescent moiety. For example, it has been shown that many raft-preferring lipids (e.g., sphingolipids and sterols), whose domain preference results from their molecular architecture, do not partition into ordered phases if fluorescently labelled, in contrast to their native counterparts. ${ }^{9}$ Incorporation of these labels into the bilayer structure may significantly perturb lipid packing, ${ }^{10}$ and labels have been reported to cause peroxidation of membrane lipids. ${ }^{11}$

Alternatively, two main types of label-free, chemically specific, high-resolution microscopy techniques have been used on lipid membranes: imaging mass spectrometry and vibrational optical microscopy. Imaging mass spectrometry ${ }^{6}$ offers very high chemical resolution, whereby biomolecular species can be distinguished and identified by accurate mass analysis. However, it is a destructive technique that works by desorbing and ionizing molecules from a sample surface. The spatial resolution is governed by the focusability of the ionizing beams and can be in the submicron scale using an energetic primary ion beam that ablates the sample surface and generates secondary ions, a process called secondary ion mass spectrometry. Nevertheless, the requirement for measurements to be performed under ultrahigh vacuum limits the application of imaging mass spectrometry to flash-frozen and freeze-dried lipid membranes, that is, membranes under nonphysiological conditions.

Vibrational optical microscopy is based on detecting the Raman-scattered light following the interaction of incident light with the intrinsic vibrational resonances of chemical bonds. It is therefore chemically specific without the need of labelling and also, in principle, noninvasive. However, spontaneous Raman scattering is a very weak process, with typical Raman scattering cross-sections of vibrating modes in organic molecules in the $10^{-29} \mathrm{~cm}^{2}$ range, resulting in very low photon fluxes of Raman-scattered light. It is therefore very challenging to detect single lipid membranes, which has led to various strategies to enhance the image contrast. One possibility is to exploit the local field enhancement effect in the vicinity of metallic tips ${ }^{12}$ (a process called tip-enhanced Raman scattering) and/or structured metallic substrates (surface-enhanced Raman scattering-SERS). ${ }^{13}$ This approach, however, requires additional sample preparation and the availability of reliable SERS substrates. Recently, Raman imaging was demonstrated on supported lipid monolayers prepared using the Langmuir-Blodgett method, ${ }^{14,15}$ which results in the lipid head groups being strongly attached to a glass substrate. Under these conditions, monolayers are extremely stable and can be imaged with high laser powers and long acquisition times. The use of Raman tags via deuterated lipids ${ }^{15}$ or lipids synthesized with a diyne moiety ${ }^{14}$ has provided additional chemical specificity. However, monolayers are not quite representative of lipid membranes, which exist as bilayers. Although lipid partitioning can be observed in lipid monolayers, the corresponding phases are different from the $L_{d}$ and $L_{o}$ phases of bilayers. Moreover, the presence of Raman tags might change lipid partitioning compared to unlabelled lipids.

Coherent Raman scattering (CRS) microscopy has emerged in the last decade as a chemically specific technique, which improves on the speed limit of spontaneous Raman microscopy, owing to the constructive interference of Raman-scattered light by identical vibrational modes coherently driven in the CRS excitation process. ${ }^{16,17}$ Briefly, CRS is a third-order nonlinearity (four-wave mixing) in which two laser fields of frequencies $\nu_{\mathrm{p}}$ (pump) and $\nu_{\mathrm{S}}$ (Stokes) coherently drive a molecular vibration in resonance at their frequency difference $\nu_{\mathrm{vib}}=\nu_{\mathrm{p}}-\nu_{\mathrm{S}}$. CRS can be detected as coherent anti-Stokes Raman scattering (CARS) or as stimulated Raman scattering (SRS). CARS is the anti-Stokes scattering of the pump field by the coherently driven vibration and occurs at the up-shifted frequency $\nu_{\mathrm{p}}+\nu_{\mathrm{vib}}=2 \nu_{\mathrm{p}}-\nu_{\mathrm{S}}$. SRS is the loss (or gain) at the pump (or Stokes) frequency from the homodyne interference of the coherent Ramanscattered field with the corresponding transmitted beam. Being a nonlinear process, CRS exhibits high spatial resolution beyond the one-photon diffraction limit and offers intrinsic optical sectioning. The coherent enhancement of CRS is particularly beneficial when imaging lipids, owing to the large number of identical $\mathrm{CH}$ bonds. Importantly, CRS is applicable to both supported and suspended lipid bilayers. Indeed, the visualization of spatially resolved lipid domains by CARS has been shown in GUVs and in supported lipid bilayers. ${ }^{18,19}$ In these works, chemical contrast was again achieved by inserting deuterated lipids and detecting them at the carbon deuterium (CD) vibrational resonance $\left(\sim 2150 \mathrm{~cm}^{-1}\right)$, which is significantly shifted compared to the nondeuterated $\mathrm{CH}$ stretch vibration $\left(\sim 2850 \mathrm{~cm}^{-1}\right)$. Deuterium labelling was implemented because CARS was excited/detected at a single vibrational resonance, which does not provide sufficient chemical specificity to distinguish lipids of different chemical compositions.

To achieve the full potential of CRS regarding its chemical sensitivity, a multiplex or hyperspectral approach must be applied, ${ }^{16,17}$ where for each spatial position, a wide spectrum of several vibrational resonances is acquired. This also enables a quantitative determination of the concentration of chemical components. ${ }^{20}$ Notably, while multiplex CARS spectroscopy was reported on supported planar lipid bilayers ${ }^{21}$ and on solutions containing unilamellar vesicles, ${ }^{22}$ spatially resolved quantitative imaging of lipid domains in single bilayers was not shown, possibly due to the slow acquisition speed of multiplex detection [utilizing a spectrometer and a charge-coupled device (CCD) camera] in these works.

We have recently developed rapid hyperspectral CARS and SRS imaging modalities based on spectral focussing of broadband femtosecond pulses, ${ }^{23,24}$ alongside a powerful quantitative data analysis method, to retrieve the spectra and concentration of chemical components. ${ }^{20,25,26}$ Here, we show the applicability of this approach to spatially resolve and chemically distinguish unlabelled $L_{o}$ and $L_{d}$ domains in singlemembrane lipid bilayers.

\section{MATERIALS AND METHODS}

Planar Lipid Bilayers. Samples were made using dioleoylphosphatidylcholine (DOPC), sphingomyelin (SM), and Chol in the molar ratios (DOPC/SM/Chol) of 1:0:0 (pure DOPC), 0:7:3 and 1:2:1 (enriched SM, forming a homogeneous $\mathrm{L}_{\mathrm{o}}$ phase), and 2:2:1 and 2:1:1 (ternary mixtures, showing coexistence of $\mathrm{L}_{\mathrm{o}}$ and $\mathrm{L}_{\mathrm{d}}$ domains at room temperature). Samples 1:2:1 and 2:1:1 were prepared using porcine SM; for all other samples, chicken-egg SM was used. All lipids were purchased from Avanti Polar Lipids (Alabaster, USA). For fluorescence labelling, we used $0.5 \mathrm{~mol} \%$ of the 
fluorescent lipid analogue 1,2-dioleoyl-sn-glycero-3-phosphoethanolamine (7-nitro-2-1,3-benzoxadiazol-4-yl) (NBD-DOPE) which partitions in the $\mathrm{L}_{\mathrm{d}}$ phase. NBD-DOPE was from SigmaAldrich (Dorset, UK). Lipid stock solutions were handled and sealed under an inert atmosphere within a nitrogen glovebox (Cole-Parmer, UK). All lipids and fluorescent lipid analogues were suspended in 2:1 chloroform/methanol (volume/ volume) or pure chloroform and stored at $-20{ }^{\circ} \mathrm{C}$ under an inert atmosphere until used.

Planar lipid bilayers were formed via osmotically induced rupture of GUVs onto a hydrophilic glass surface (mixtures 1:0:0, 1:2:1, 2:1:1, and 2:2:1) or via spin-coating (mixtures 1:0:0 and 0:7:3). Glass coverslips were cleaned with acetone to remove inorganic contaminants. They were then submerged in $60 \mathrm{~mL}$ of sulfuric acid at $95{ }^{\circ} \mathrm{C}$. $20 \mathrm{~mL}$ of hydrogen peroxide was added to the acid after several minutes. The mixture was allowed to react for $1 \mathrm{~h}$, after which the coverslips were washed with distilled water and finally dried under a stream of nitrogen. The etching with acid and hydrogen peroxide served to remove leftover organic contaminants and increase the hydrophilicity of the coverslip surface. Etched coverslips were stored in nitrogen at around $5{ }^{\circ} \mathrm{C}$ in order to preserve the hydrophilicity.

GUVs were created using an electroformation protocol published previously. ${ }^{8}$ Briefly, $10 \mu \mathrm{L}$ of lipid solution ( $1 \mathrm{~g} / \mathrm{L}$ ) was spread onto the surface of two tantalum electrodes, followed by removal of the solvent for $1 \mathrm{~h}$ under high vacuum. The electrodes were then suspended in $550 \mu \mathrm{L}$ of deionized water at $60{ }^{\circ} \mathrm{C}$ and subjected to a $1.2 \mathrm{~V}$ peak-to-peak square waveform at $10 \mathrm{~Hz}$ for $1 \mathrm{~h}$ to induce GUV formation. Subsequently, the voltage was increased to $1.5 \mathrm{~V}$ peak-to-peak with a sinusoidal waveform, and the frequency was reduced to $5 \mathrm{~Hz}$ for $30 \mathrm{~min}$, then $2 \mathrm{~Hz}$ for $15 \mathrm{~min}$, and finally $1 \mathrm{~Hz}$ for 15 min to encourage the GUVs to go into solution. For GUVs forming lipid domains, the chamber was sealed, and cooling to room temperature was controlled in steps of $4{ }^{\circ} \mathrm{C} / \mathrm{h}$ to control domain formation. A thin imaging spacer $(120 \mu \mathrm{m}$ thick with a $20 \mathrm{~mm}$ diameter opening, Grace Biolabs) was attached to the treated glass coverslip surface prior to deposition of and incubation with $8 \mu \mathrm{L}$ of the aforementioned GUV solution for $15 \mathrm{~min}$. Following incubation, $8 \mu \mathrm{L}$ of $75 \mathrm{mM}$ phosphatebuffered saline (PBS) was added to induce GUV rupture, and the chamber was immediately sealed with a clean glass slide. Preparation of the 2:2:1 sample was slightly different, with the last step involving deposition of $65 \mu \mathrm{L}$ (rather than $8 \mu \mathrm{L}$ ) of GUV solution, which was topped up with another $65 \mu \mathrm{L}$ after 5,15 , and $25 \mathrm{~min} ; 65 \mu \mathrm{L}$ of PBS was then added, and $65 \mu \mathrm{L}$ of the medium was exchanged with $65 \mu \mathrm{L}$ of PBS 10 times to remove any free-floating GUVs and other lipid debris; this was all done at $55^{\circ} \mathrm{C}$.

When using spin-coating, $150 \mu \mathrm{L}$ of the mixture was spun on an etched coverslip at $3000 \mathrm{rpm}$ with a $6 \mathrm{~s}$ constant acceleration and deceleration on either side of a $30 \mathrm{~s}$ period for a total of $42 \mathrm{~s}$. The coverslip was then placed in a plastic centrifuge tube with a small piece of wet tissue. The tube was filled with nitrogen to prevent lipid peroxidation, sealed, and incubated in an oven at $37^{\circ} \mathrm{C}$ for $1 \mathrm{~h}$. An imaging spacer was then placed on the lipid-containing side of the coverslip to form a cylindrical well at the bottom of which the lipid sat. This was filled with degassed PBS. Multilamellar (multiplebilayer) versions of the 0:7:3 and 1:0:0 samples were also made following the same procedure as above but using 20 times more lipid in the solution.
Optical Microspectroscopy. Epi-fluorescence and differential interference contrast (DIC) microscopy was performed on an Eclipse Ti-U Nikon microscope stand as described in our previous works. ${ }^{7,8}$ Here, we used a $60 \times 1.27$-NA waterimmersion objective and a 1.34-NA oil-immersion condenser. DIC and fluorescence images were acquired using a CCD camera (Orca 285, Hamamatsu, Japan). DIC illumination was provided by a halogen tungsten lamp (V2-A LL 100W, Nikon) followed by a blue-green filter (BG40, Schott) to block nearinfrared light, for which the DIC polarizers do not have sufficient extinction, and a green filter [GIF, transmission band $(550 \pm 20) \mathrm{nm}$; Nikon] to define the wavelength range for the quantitative differential interference contrast (qDIC) analysis. For the measurements shown on the DOPC/SM/Chol 2:1:1 sample, an exposure time of $0.1 \mathrm{~s}$ was used for each frame in the DIC images. The average over 512 frames was acquired for each angle of the polarizer (offset phase). For more details, see the Supporting Information. Fluorescence images were acquired with an exposure time of $2.5 \mathrm{~s}$. Epi-fluorescence excitation was performed using a metal-halide lamp (Lumen L200/D, Prior Scientific, USA) at $10 \%$ power and an exciter/ emitter/dichroic filter cube (GFP-A-Basic; Semrock, USA) suitable for the NBD dye.

Confocal Raman spectra of bulk lipids were taken using the Ti-U Nikon microscope stand equipped with a $20 \times 0.75-\mathrm{NA}$ objective. A $532 \mathrm{~nm}$ continuous-wave laser excitation was filtered with a Semrock laser line filter (LL01-532) and coupled into the microscope by a dichroic mirror (Semrock LPD01-532RS) with a power of $10 \mathrm{~mW}$ at the sample. Raman scattering was collected in the epi direction, filtered with a long-pass filter (Semrock BLP01-532R), dispersed by an imaging spectrometer (HORIBA iHR550) with a 600 lines/ $\mathrm{mm}$ grating, and detected with a CCD camera (Andor Newton DU971N-BV) with a full width at half-maximum (fwhm) spectral resolution of about $2 \mathrm{~cm}^{-1}$.

CARS was measured using the setup and technique described in our previous work ${ }^{23}$ with a $60 \times 1.27$-NA waterimmersion objective and a 1.34-NA oil-immersion condenser (see the Supporting Information for more details). It consists of a home-built microscope with the same Ti-U Nikon microscope stand described above, using a single-broadband 5 fs pulsed Ti:Sa laser source (Venteon, Pulse:One PE) to provide the pump and Stokes beams for CARS, as well as a third beam for two-photon fluorescence (TPF). The pulses are centered at 685 or $689 \mathrm{~nm}$ (pump) and $806 \mathrm{~nm}$ (Stokes), with a bandwidth at $10 \%$ intensity of 65 and $200 \mathrm{~nm}$, respectively. They are linearly chirped using glass blocks, and their delay time is tuned in order to drive vibrational resonances in the 2700-3100 $\mathrm{cm}^{-1}$ wave number range with a spectral resolution of about $10 \mathrm{~cm}^{-1}$, a technique known as spectral focussing. ${ }^{27}$ TPF excitation is centered at $940 \mathrm{~nm}$, which is suitable to excite the NBD dye, with a Fourier-limited pulse duration of approximately $30 \mathrm{fs}$ at the sample. CARS and TPF are collected in the forward direction by the condenser lens, separated using appropriate filters, and detected using photomultiplier tubes as described in our previous work. ${ }^{23} \mathrm{~A}$ pixel dwell time of $10 \mu \mathrm{s}$ was used for all CARS and TPF measurements. Typical pump, Stokes, and TPF excitation powers at the sample were 40,20 , and $25 \mathrm{~mW}$, respectively. Frame averaging was used as indicated in the figure captions. For correlative TPF/CARS imaging, TPF was conducted first; subsequently, the dye was fully photobleached using the CARS pump beam (typically, 10 rasterscans across the sample were 
sufficient to remove all residual fluorescence). In this way, CARS imaging was free from fluorescence artefacts. Backgrounds were measured under the same excitation and detection conditions with pump and Stokes pulses out of time overlap and were subtracted from the measured CARS intensities.

SRS imaging was performed using the setup described in detail in our previous work. ${ }^{24}$ Briefly, a pulsed Ti:Sa laser (Spectra-Physics Mai Tai) emitting 150 fs pulses centered at $820 \mathrm{~nm}$ is used as the pump beam for stimulated Raman loss (SRL). An optical parametric oscillator pumped by the second harmonic of the Ti:Sa laser (Radiantis Inspire) provides the Stokes beam. Here, its wavelength was set to $1070 \mathrm{~nm}$, resulting in a central wave number of $2850 \mathrm{~cm}^{-1}$. A spectral range of $2700-3100 \mathrm{~cm}^{-1}$ was scanned by spectral focussing as described above, with a spectral resolution of about $30 \mathrm{~cm}^{-1}$. The sample was mounted on a Nikon Ti-U microscope with a $60 \times 1.27-N A$ water-immersion objective (Nikon MRD70650) and a $1.5 \times$ tube lens. For SRL detection, the Stokes beam was amplitude-modulated by an acousto-optic modulator using a $2.5 \mathrm{MHz}$ square wave, and the resulting modulation of the pump beam in transmission, collected by a 1.34-NA condenser lens, was measured using a silicon photodiode and a lock-in amplifier. In this setup, forward CARS can be detected simultaneously with SRL using a photomultiplier. ${ }^{24}$ At the sample, the pump power was about $5 \mathrm{~mW}$ and the Stokes power was about $11 \mathrm{~mW}$. A pixel dwell time of $1 \mathrm{~ms}$ was used without frame averaging.

All samples were imaged using DIC to identify the regions of interest and to check for photodamage, which was not observed under the indicated excitation and detection conditions. An example comparing DIC acquired before and after SRS imaging is shown in Supporting Information, Section 3.i. When performing long acquisitions for hyperspectral imaging, stability of the image focal plane and of the sample was monitored by repeating the in-plane image acquisition at $2850 \mathrm{~cm}^{-1}$ before and after the hyperspectral scan and verifying its reproducibility. Note that CARS hyperspectral datasets were acquired as averages over 10 frames as this repetition was observed to be within the stability and reproducibility limits on all investigated samples.

\section{RESULTS}

CARS Microspectroscopy of a Single Lipid Bilayer. To quantify the CARS signal strength and chemical contrast, we compare the CARS spectrum measured on a single planar lipid bilayer consisting of only DOPC with that of bulk DOPC in the $\mathrm{CH}$ stretch vibrational range, as shown in Figure 1. For this experiment, we fabricated supported planar lipid bilayers through osmotically induced rupture of GUVs onto a hydrophilic glass surface (see Materials and Methods). CARS microspectroscopy was performed with $10 \mathrm{~cm}^{-1}$ resolution via spectral focusing (see Materials and Methods). xyz images were acquired to locate the bilayer in the optimum focal plane, and a series of $x y$ images at wave numbers of $2700-3100 \mathrm{~cm}^{-1}$ was recorded to resolve the lipid vibrational resonances. The resulting hyperspectral CARS datasets were denoised and corrected from a spatially varying background (using a polynomial fit) to account for slight changes in the spatial overlap between pump and Stokes beams and/or sample tilt over the scan range (for details, see the Supporting Information). The measured intensity at the bilayer (spatially averaged) was then divided by the nonresonant CARS

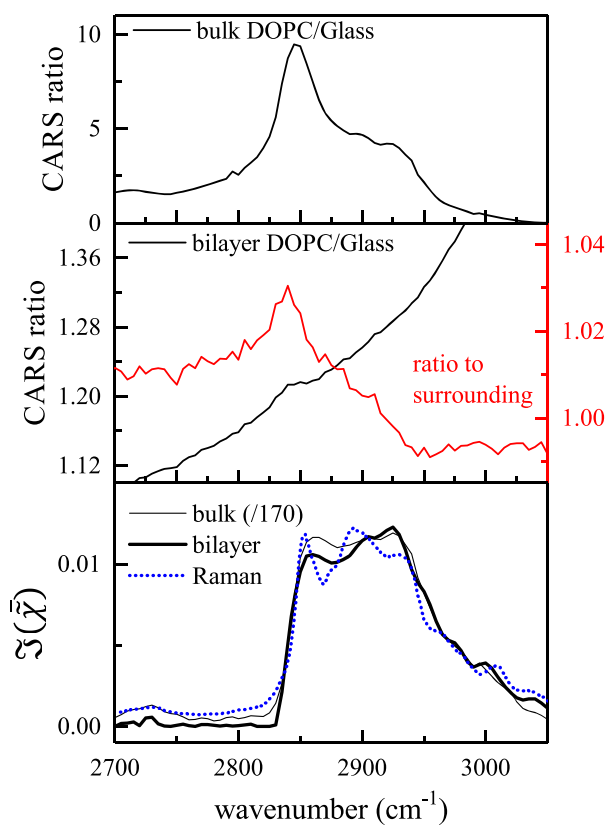

Figure 1. Top: Spectrum of CARS intensity ratio between bulk DOPC and the glass coverslip support. Center: Spectrum of CARS intensity ratio between a homogeneous DOPC lipid bilayer and either glass (black) or the surrounding aqueous region (red). Bottom: Retrieved imaginary part of the CARS susceptibility $\mathfrak{\Im}(\overline{\widetilde{\chi}})$ for bulk DOPC and the bilayer (mean over 5 bilayer samples, see main text). The measured Raman spectrum of bulk DOPC is shown for comparison (blue dotted curve). CARS spectra in the lipid bilayer were obtained from spatially averaged hyperspectral images of about $15 \mu \mathrm{m} \times 15 \mu \mathrm{m}$ measured using a $60 \times 1.27-\mathrm{NA}$ water-immersion objective, a pixel dwell time of $0.01 \mathrm{~ms}$, a pixel size of $0.1 \mu \mathrm{m}$, a 10 frame average, and a laser power of $40 \mathrm{~mW}$ (pump) and $20 \mathrm{~mW}$ (Stokes) at the sample.

intensity measured in the same image from a surrounding region without the bilayer (also spatially averaged) under the same excitation and detection conditions in order to correct for the varying temporal overlap of the pump and Stokes beams and to derive a CARS intensity ratio independent of excitation/detection parameters. The resulting spectrum is shown in Figure 1 (center) and exhibits the characteristic dispersive line shape from the interference between the resonant and nonresonant parts of the CARS susceptibility, which is expected when the resonant material fills only a small fraction of the focal volume, as is the case for a lipid bilayer. ${ }^{21}$ The CARS intensity ratio relative to the nonresonant CARS background measured in the glass coverslip is also shown, with the dispersive lipid resonance being superimposed onto the response from the surround aqueous buffer (PBS). Note that the amplitude of the dispersive lipid resonance is only a small fraction $(\sim 3 \%)$ of the signal. The CARS intensity ratio relative to glass, measured in bulk DOPC, is given in the top frame for comparison and shows a much larger amplitude, as expected considering the small thickness of the bilayer compared to the size of the axial point-spread function (PSF). From the measured CARS ratio, the imaginary part of the CARS susceptibility, $\mathfrak{I}(\overline{\widetilde{\chi}})$, is retrieved using a phase-corrected Kramers-Kronig (PCKK) procedure, ${ }^{20}$ which recovers a resonant Raman-like spectrum. The bottom panel of Figure 1 shows the $\mathfrak{\Im}(\overline{\widetilde{\chi}})$ retrieved for bulk DOPC together with the measured Raman spectrum. For the bilayer, we further applied 
a factorization procedure ${ }^{20,28}$ (called FSC ${ }^{3}$ ) in order to remove residual artefacts at the glass-PBS interface (see also the Supporting Information). The spatially averaged spectrum in the bilayer region of the corresponding component is deduced. This is shown for comparison in the bottom panel of Figure 1 as the mean spectrum for five nominally identical bilayer samples. Notably, the retrieved $\mathfrak{I}(\overline{\widetilde{\chi}})$ spectra of both bulk DOPC and the bilayer greatly resemble the Raman spectrum of DOPC, but the amplitude of $\mathfrak{J}(\overline{\widetilde{\chi}})$ in bulk DOPC is higher than that in the bilayer by a factor of 170 . Since $\mathfrak{I}(\overline{\widetilde{\chi}})$ is linear in the number of chemical bonds in the focal volume, this factor reflects the ratio between the bilayer thickness and the effective axial extension of the PSF. Considering a bilayer thickness of about $5 \mathrm{~nm}$, this extension is estimated to be 170 $\times 5=850 \mathrm{~nm}$, consistent with the measured axial PSF of 0.9 $\mu \mathrm{m}$ fwhm for the CARS field in the setup used. ${ }^{29}$ It is also useful to estimate the number of DOPC molecules generating the measured CARS for the bilayer, taking into account the lateral PSF extension of $0.3 \mu \mathrm{m}$ fwhm for the CARS field ${ }^{29}$ and the area $A$ per DOPC molecule in the bilayer. Using $A=70.15$ $\AA^{2}$, as reported in the literature at room temperature, ${ }^{30}$ we estimate $2 \times 10^{5}$ DOPC molecules contributing to the measured CARS signal. Note that, under the excitation and detection conditions indicated in Figure 1, the signal-to-noise ratio observed in each pixel of the CARS image of the bilayer (i.e., without spatial averaging) was about 1 (see the Supporting Information S1). From this, we deduce a sensitivity limit of about 2000 DOPC molecules $/ \sqrt{\mathrm{Hz}}$ for the power and focussing conditions used in Figure 1.

Fluorescence Microscopy and DIC of Lipid Domains. First, we demonstrated domain formation using conventional fluorescence microscopy. An example of a single-membrane planar lipid bilayer containing lipid domains is shown in Figure 2. We used a ternary mixture of DOPC, SM, and Chol in a 2:1:1 molar ratio, which is known to form $\mathrm{L}_{\mathrm{o}}$ and $\mathrm{L}_{\mathrm{d}}$ domains
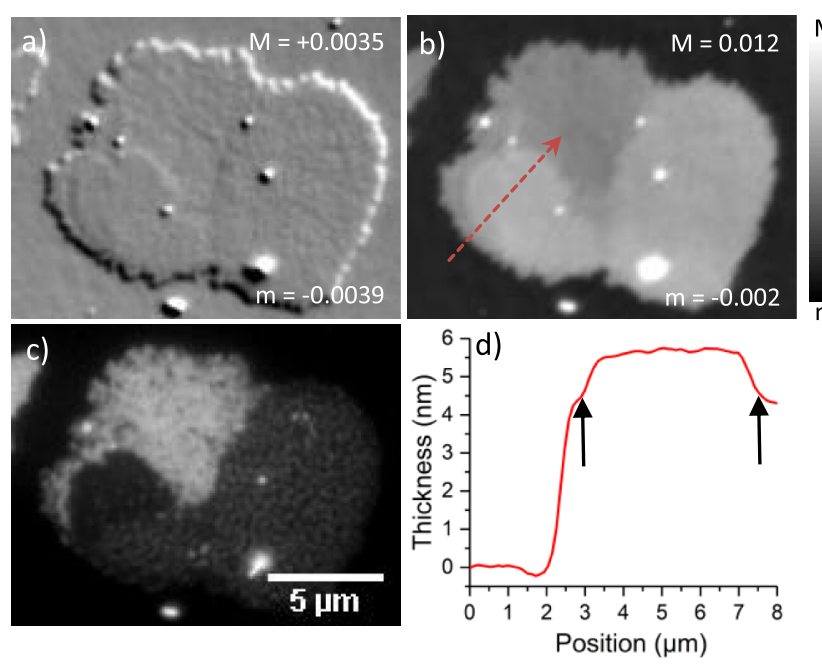

Figure 2. (a) DIC differential phase $(\delta)$ image of a planar lipid bilayer with a ternary mixture of DOPC/SM/Chol in a 2:1:1 molar ratio exhibiting $\mathrm{L}_{\mathrm{o}}$ and $\mathrm{L}_{\mathrm{d}}$ domains. (b) Corresponding integrated phase $(\varphi)$ image. (c) Epi-fluorescence image of the bilayer labelled with the fluorescent lipid analogue NBD-DOPE, which partitions in the $\mathrm{L}_{d}$ phase. (d) Thickness profile across the red line in (b), with transitions from the thinner $L_{d}$ phase to the thicker $L_{o}$ phase indicated by the arrows. at room temperature. ${ }^{31}$ For fluorescence labelling, we used a small percentage $(0.5 \mathrm{~mol} \%)$ of the fluorescent lipid analogue NBD-DOPE, which partitions in the $L_{d}$ phase. ${ }^{32}$ An epifluorescence image of the lipid bilayer exhibiting one bright $\mathrm{L}_{d}$ microdomain is shown in Figure 2c.

DIC microscopy measures the difference of the optical phase between two points in the sample plane spatially separated by an amount (the shear) typically comparable with the optical resolution. Based on this principle, we have developed a qDIC image acquisition and analysis procedure to measure the spatial distribution of the optical phase, ${ }^{8,33}$ which for lipid bilayers of known refractive index can in turn be used to calculate their thickness. ${ }^{7}$ Briefly, we start by acquiring a contrast image defined as

$$
I_{c}=\frac{I_{+}-I_{-}}{I_{+}+I_{-}}
$$

where $I_{ \pm}$are the transmitted intensities for opposite angles of the polarizer in a DIC setup with a de Sénarmont compensator. ${ }^{8}$ The differential phase is defined as $\delta=\varphi_{+}-$ $\varphi_{-}$, where

$$
\varphi_{ \pm}=\varphi\left(\mathbf{r} \pm \frac{\mathbf{s}}{2}\right)
$$

is the optical phase accumulated in the sample for the beam passing through the point $\mathbf{r} \pm \mathbf{s} / 2$, $\mathbf{s}$ is the shear vector, and $\mathbf{r}$ is the DIC image coordinate on the sample plane. We calculate $\delta$ using the exact analytical solution of the relationship between $I_{c}$ and $\delta$ (see the Supporting Information). The spatial distribution of the optical phase at the sample, $\varphi(\mathbf{r})$, is then calculated from $\delta$ by performing a Wiener deconvolution procedure, ${ }^{8}$ further optimized to reduce integration artefacts as discussed in our recent work. ${ }^{7}$ In Figure 2a, we show the qDIC $\delta$ image of the bilayer. The corresponding integrated optical phase image is shown in Figure $2 \mathrm{~b}$. Notably, $\varphi(\mathbf{r})$ correlates with the epi-fluorescence image as an inverted contrast, which is expected because $L_{o}$ domains are thicker than $L_{d}$ domains. The bilayer thickness $t$ can be calculated taking into account that the optical phase introduced by the lipid bilayer is

$$
\varphi(\mathbf{r})=\frac{2 \pi \Delta_{n}}{\lambda_{0}} t(\mathbf{r})
$$

where $\lambda_{0}$ is the wavelength in vacuum, $\Delta_{n}$ is the refractive index change between the lipid bilayer and its surrounding aqueous medium, and $t(\mathbf{r})$ is the thickness profile of the bilayer. Using $\Delta_{n}=0.1159$ under the experimental condition $\lambda_{0}=550 \mathrm{~nm}$, we obtain the thickness profile shown in Figure $2 \mathrm{~d}$ along the red line in Figure $2 \mathrm{~b}$. From this profile, we find a thickness of about $4 \mathrm{~nm}$ for the bilayer in the $\mathrm{L}_{\mathrm{d}}$ domain, in good agreement with the thickness of a DOPC-only bilayer measured by others using ellipsometry, ${ }^{34}$ and an increase of about $1 \mathrm{~nm}$ going from the $\mathrm{L}_{\mathrm{d}}$ phase to the $\mathrm{L}_{\mathrm{o}}$ phase, also in agreement with findings from AFM studies ${ }^{35}$ and our recent qDIC work. ${ }^{7}$ Because of the measurement of the differential phase in DIC, thickness steps at the boundaries between domains can be reliably inferred, but absolute thicknesses over large distances carry increasing systematic errors. Even with these limitations, qDIC is a remarkably sensitive label-free optical method for distinguishing lipid domains based on their optical phase differences, as shown in our recent work where the accuracy and precision of the method are discussed in detail. $^{7}$ 

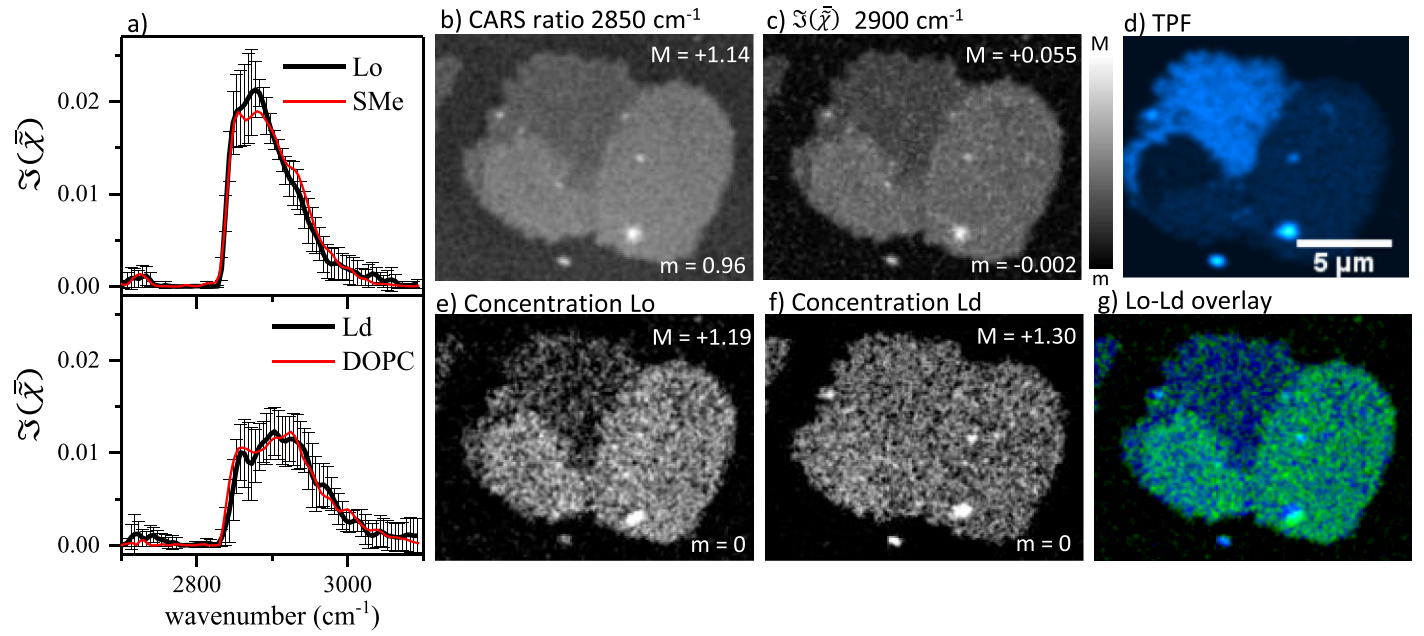

Figure 3. Hyperspectral CARS imaging of the planar bilayer in Figure 2 exhibiting $L_{o}$ and $L_{d}$ domains. (a) Spectra obtained using an unsupervised factorization into chemical components on nine nominally equal bilayer patches exhibiting $\mathrm{L}_{\mathrm{o}}$ and $\mathrm{L}_{\mathrm{d}}$ domains as mean (solid line) and standard deviation (bar). Red lines are spectra (mean from five nominally equal samples) measured on homogeneous bilayers containing a DOPC/SM/Chol = 1:2:1 mixture forming a homogeneous $\mathrm{L}_{\mathrm{o}}$ phase (called SMe) or DOPC only. (b) CARS intensity relative to the spatially averaged intensity in the region surrounding the bilayer, imaged at $2850 \mathrm{~cm}^{-1}$. (c) Retrieved CARS susceptibility $\Im(\overline{\widetilde{\chi}})$ at $2900 \mathrm{~cm}^{-1}$. (d) TPF image of the bilayer labelled with the fluorescent lipid analogue NBD-DOPE partitioning in the $\mathrm{L}_{d}$ phase. $(\mathrm{e}, \mathrm{f})$ Spatial distribution of the $\mathrm{L}_{\mathrm{o}}$ and $\mathrm{L}_{\mathrm{d}}$ concentration components. Gray scales are from $\mathrm{m}$ to $\mathrm{M}$. (g) Color overlay of $\mathrm{L}_{\mathrm{d}}$ (blue) and $\mathrm{L}_{\mathrm{o}}$ (green). CARS measurements were performed using a $0.01 \mathrm{~ms}$ pixel dwell time, $0.1 \mu \mathrm{m}$ pixel size, 10 -frame average, and laser powers of $40 \mathrm{~mW}$ (pump) and $20 \mathrm{~mW}$ (Stokes) at the sample. For TPF, the excitation power was 25 $\mathrm{mW}$ at the sample, and 20 -frame averaging was used.

Chemically Specific Hyperspectral CARS Imaging of Lipid Domains. We then performed hyperspectral CARS imaging on the planar single bilayer shown in Figure 2. Here, the entire hyperspectral image stack as a series of $x y$ images at different frequencies in the $2700-3100 \mathrm{~cm}^{-1}$ range is analyzed using the PCKK phase retrieval and $\mathrm{FSC}^{3}$ factorization methods described previously. ${ }^{20}$ The spatially resolved distribution of separated chemical components is obtained together with the corresponding $\mathfrak{\Im}(\overline{\widetilde{\chi}})$ spectra. Importantly, the analysis is unsupervised, that is, it does not require prior knowledge of the sample's chemical composition. We find that the method is able to distinguish two main chemical components, as shown in Figure 3. The spatial distribution of the component concentrations (Figure 3e,f) correlates very well with the lipid domains in Figure 2. It also correlates with the intensity distribution of the single-frequency CARS image (as intensity ratio to the surrounding) taken at the $\mathrm{CH}_{2}$ symmetric stretch resonance, $2850 \mathrm{~cm}^{-1}$, shown in Figure $3 \mathrm{~b}$, which is expected on the basis of the molecular packing density difference between $L_{o}$ and $L_{d}$ domains. ${ }^{36}$ Figure $3 c$ shows the phase-retrieved CARS susceptibility at $2900 \mathrm{~cm}^{-1}$, where maximum contrast of the domains in $\Im(\overline{\widetilde{\chi}})$ is obtained. Figure $3 \mathrm{~d}$ is the TPF image of the bilayer labelled with the fluorescent lipid analogue NBD-DOPE, which partitions in the $\mathrm{L}_{\mathrm{d}}$ phase. The retrieved $\mathfrak{\Im}(\overline{\widetilde{\chi}})$ factorized spectra of the two chemical components are shown in Figure 3a. They are compared with reference spectra measured on homogeneous planar bilayers containing either only DOPC (the main component of the $\mathrm{L}_{\mathrm{d}}$ phase) or a SM-enriched DOPC/SM/ Chol $=1: 2: 1$ mixture forming a homogeneous $L_{o}$ phase. Reference spectra are shown as spatial averages over the bilayer region (as discussed for Figure 1), and their amplitude corresponds to having a spatially averaged concentration of 1 . For the spectra of the two chemical components, amplitudes are calculated such that the spectral integral is equal to that of the corresponding reference spectrum. Within the distribution from measurements repeated on nominally identical samples, we find a very good correspondence between the spectra retrieved from the unsupervised analysis in the bilayers containing coexisting lipid domains (using the 2:1:1 mixture) and the reference spectra from homogeneous bilayers, clearly showing the ability of the technique to chemically resolve $L_{o}$ and $L_{d}$ domains. We note the differences between the $\mathfrak{J}(\overline{\widetilde{\chi}})$ spectrum in the $\mathrm{L}_{\mathrm{o}}$ phase, which is narrower and more dominated by the $\mathrm{CH}_{2}$ symmetric stretch vibration peak at $2850 \mathrm{~cm}^{-1}$, and the spectrum in the $\mathrm{L}_{\mathrm{d}}$ phase, which is broader with a pronounced shoulder around $2930 \mathrm{~cm}^{-1}$ due to a combination of $\mathrm{CH}_{3}$ stretch vibrations and $\mathrm{CH}_{2}$ asymmetric stretch enhanced by the broadening and shift of the $\mathrm{CH}$ deformations in the liquid phase. These differences are consistent with previous knowledge from Raman and CARS spectroscopy of saturated, more orderly packed lipids versus unsaturated, disorderly packed lipids. ${ }^{21,37,38}$ Notably, the Raman spectrum of pure SM reported in the literature ${ }^{39}$ (also measured by us, see Supporting Information S16) exhibits a strong sharp peak near $2880 \mathrm{~cm}^{-1}$ not observed in the $\Im(\overline{\widetilde{\chi}})$ spectrum of the $\mathrm{L}_{\mathrm{o}}$ phase. This is because pure SM at room temperature is in the gel (solid) phase. Indeed, to obtain the $\mathrm{L}_{\mathrm{o}}$ phase, SM has to be mixed with Chol. ${ }^{31}$ On the other hand, the Raman spectrum of pure Chol has a dominant peak near $2930 \mathrm{~cm}^{-1}$ from the $\mathrm{CH}_{3}$ symmetric stretch bonds $\mathrm{s}^{39}$ (see Supporting Information S16), while in the $\mathfrak{\Im}(\overline{\widetilde{\chi}})$ spectrum of the $L_{o}$ phase, the $2930 \mathrm{~cm}^{-1}$ band appears as a shoulder of lower amplitude compared to the dominant $\mathrm{CH}_{2}$ peak at $2850 \mathrm{~cm}^{-1}$. This can be understood considering that a Chol molecule has only $5 \mathrm{CH}_{3}$ groups, compared to the long acyl chain with more than $16 \mathrm{CH}_{2}$ bonds in SM, and that the $\mathrm{L}_{\mathrm{o}}$ phase is enriched in SM with an equilibrium stoichiometry $\mathrm{SM} / \mathrm{Chol}$ reported $^{40}$ to be near $2: 1$. 


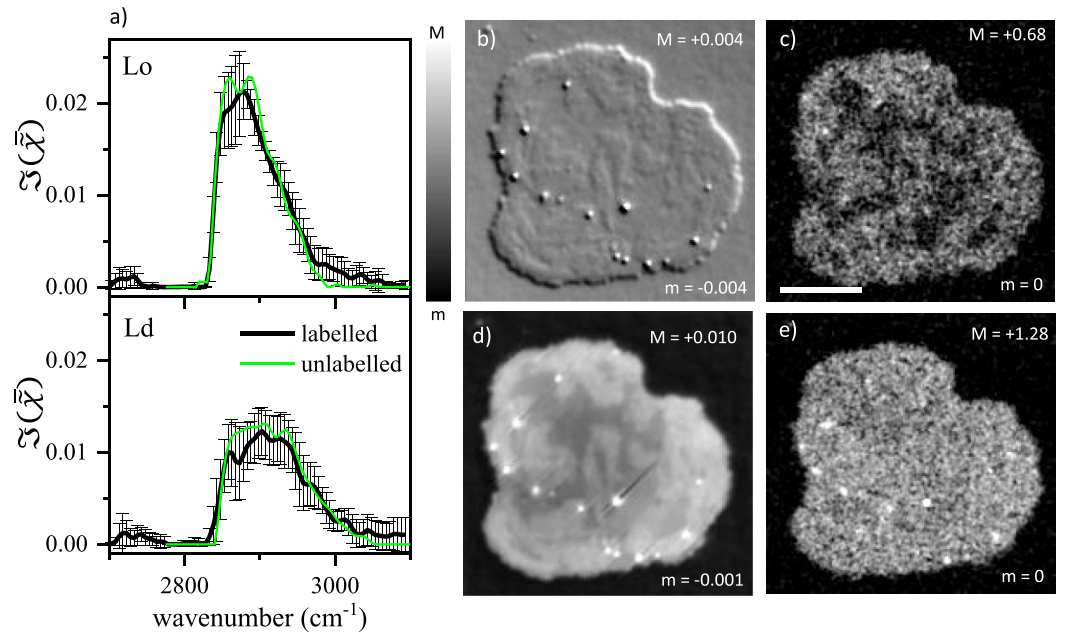

Figure 4. CARS hyperspectral imaging on unlabelled lipid bilayers exhibiting $L_{o}$ and $L_{d}$ domains. (a) Unsupervised factorization into chemical components corresponding to the $\mathrm{L}_{\mathrm{o}}$ and $\mathrm{L}_{\mathrm{d}}$ domains. Spectra are obtained using global factorization on eight nominally identical unlabelled bilayers and compared to the spectra in the nine labelled samples. The spatial distribution of the concentrations is shown in $(c)\left(\mathrm{L}_{\mathrm{o}}\right)$ and $(\mathrm{e})\left(\mathrm{L}_{\mathrm{d}}\right)$. (b) DIC differential phase image of one of the lipid bilayers. (d) Optical phase image of the same bilayer. Scale bar: $5 \mu \mathrm{m}$. Gray scales are from $\mathrm{m}$ to M. CARS measurements were performed using a $0.01 \mathrm{~ms}$ pixel dwell time, $0.1 \mu \mathrm{m}$ pixel size, 10 -frame average, and laser powers of $50 \mathrm{~mW}$ (pump) and $30 \mathrm{~mW}$ (Stokes) at the sample.
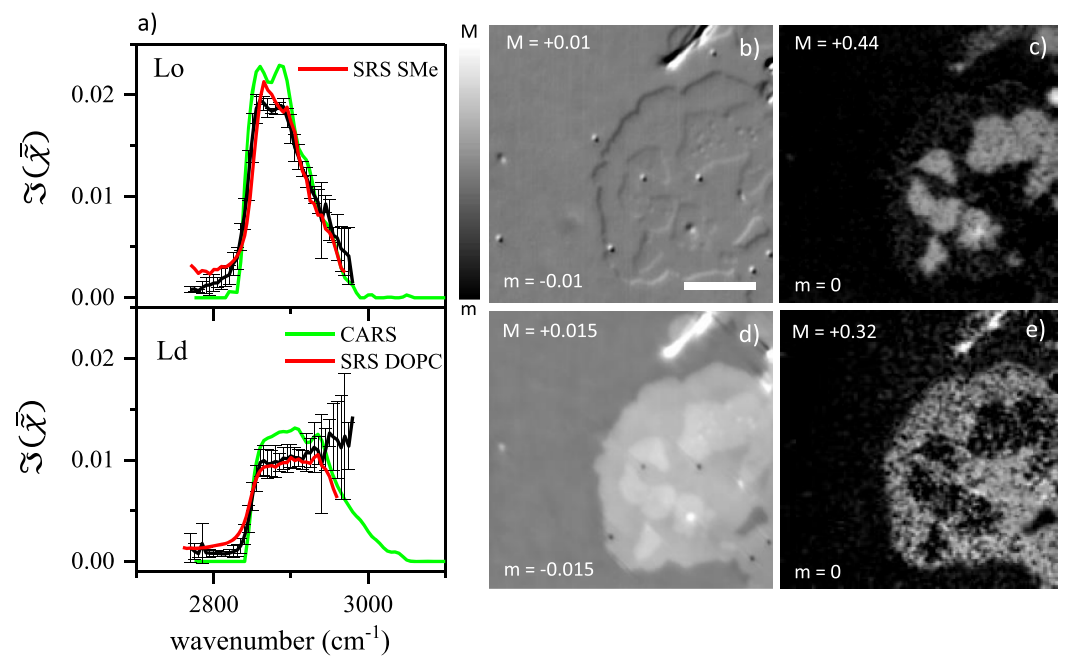

Figure 5. SRL hyperspectral imaging on label-free planar lipid bilayers made of a ternary mixture of DOPC/SM/Chol in a 2:2:1 molar ratio exhibiting $\mathrm{L}_{\mathrm{o}}$ and $\mathrm{L}_{d}$ domains. (a) Unsupervised factorization into chemical components corresponding to the $\mathrm{L}_{\mathrm{o}}$ and $\mathrm{L}_{\mathrm{d}}$ domains. Spectra are from five nominally identical unlabelled bilayers, shown as mean (solid curve) and standard deviation (bar). Red curves are reference SRL spectra measured on either an SMe bilayer or a pure DOPC homogeneous layer, as indicated. Green curves are the CARS spectra of the unlabelled domains from Figure 4. An example of the spatial distribution of the concentrations corresponding to the SRL spectra of the domains is shown in (c) $\left(\mathrm{L}_{\mathrm{o}}\right)$ and $(\mathrm{e})\left(\mathrm{L}_{\mathrm{d}}\right)$. (b) DIC differential phase image of one of the lipid bilayers. (d) Optical phase image of the same bilayer. Scale bar: $5 \mu \mathrm{m}$. Gray scales are from $\mathrm{m}$ to M. SRL measurements were performed using a pixel dwell time of $1 \mathrm{~ms}$, a pixel size of $0.072 \mu \mathrm{m}$, and a laser power of 5 $\mathrm{mW}$ (pump) and $11 \mathrm{~mW}$ (Stokes) at the sample.

Having demonstrated that hyperspectral CARS microscopy analyzed with our unsupervised factorization procedure is able to separate $\mathrm{L}_{\mathrm{o}}$ and $\mathrm{L}_{\mathrm{d}}$ domains spatially and spectrally in single lipid bilayers, we then tested the method on label-free bilayers. Figure 4 shows the results on eight nominally identical unlabelled bilayers exhibiting $L_{o}$ and $L_{d}$ domains. In this case, we performed an unsupervised "global" factorization where we considered spectral components common to all images rather than an individual analysis for each sample separately, as was done to obtain the results in Figure 3. A global analysis was needed because the spatial separation between $\mathrm{L}_{\mathrm{o}}$ and $\mathrm{L}_{\mathrm{d}}$ domains was generally less marked in the unlabelled bilayers, such that, in some cases, an individual unsupervised analysis was unable to return separate domains. The retrieved $\mathfrak{\Im}(\overline{\widetilde{\chi}})$ factorized global spectra of the two chemical components corresponding to $\mathrm{L}_{\mathrm{o}}$ and $\mathrm{L}_{\mathrm{d}}$ are shown in Figure $4 \mathrm{a}$ and compared with the spectra of the labelled ternary bilayers from Figure 3a. Here, amplitudes are calculated such that the spectral integral is equal to that of the corresponding homogeneous bilayer mean spectrum shown in Figure 3a. Within errors, we observe a good agreement between the spectral components of the labelled samples and those of the unlabelled ones. An example of the corresponding spatial distributions of the component concentrations is shown in Figure $4 c$,e and compares well with the spatial profile measured in $\mathrm{qDIC}$, as shown in Figure $4 \mathrm{~b}, \mathrm{~d}$. As mentioned, the spatial 
separation between $\mathrm{L}_{\mathrm{o}}$ and $\mathrm{L}_{\mathrm{d}}$ domains appeared less pronounced in the unlabelled bilayers when compared to the labelled bilayers across all investigated samples (see Supporting Information S6 and S7 for an overview). This suggests that the inclusion of the dye, while not altering the spectra, influences the spatial separation of the domains.

SRS Hyperspectral Imaging of Lipid Domains. After having shown that CARS is able to image $\mathrm{L}_{\mathrm{o}}$ and $\mathrm{L}_{\mathrm{d}}$ domains and determine their $\mathfrak{\Im}(\overline{\widetilde{\chi}})$ spectra, we investigated label-free single lipid bilayers by SRS hyperspectral microscopy. We used the ternary mixture 2:2:1 as this resulted in spatially wellseparated $\mathrm{L}_{\mathrm{o}}$ and $\mathrm{L}_{\mathrm{d}}$ domains. SRS was measured in the form of SRL. Our setup enabled simultaneous acquisition of forwardtransmitted CARS intensity (see Materials and Methods). As was done for the CARS data sets, measured hyperspectral SRL data sets were first denoised and corrected from a spatially varying background (using a polynomial fit) to account for slight changes in the spatial overlap between pump and Stokes beams and/or sample tilt over the scan range (for details see the Supporting Information). The aforementioned unsupervised $\mathrm{FSC}^{3}$ procedure was then applied to retrieve the spectra and concentration maps of the $\mathrm{L}_{\mathrm{o}}$ and $\mathrm{L}_{\mathrm{d}}$ components. SRL spectra are not affected by vibrationally nonresonant background and are thus Raman-like; therefore, it is not necessary to apply the PCKK field-retrieval procedure which is used for the CARS ratio. ${ }^{20}$

In order to correct the SRL spectra for the varying pumpStokes pulse overlap during spectral focusing, we used the CARS intensity, which is dominated by the nonresonant background and measured simultaneously to the SRL. We note that the CARS intensity is proportional to $\left.\left|E_{\mathrm{p}}{ }^{4}\right| E_{\mathrm{s}}\right|^{2}$, where $E_{\mathrm{p}}$ is the pump field and $E_{\mathrm{s}}$ is the Stokes field, while the SRL intensity is proportional to $\left|E_{\mathrm{p}}\right|^{2}\left|E_{\mathrm{s}}\right|$. For the correction, we therefore divide the SRL signal by the square root of the spatially averaged CARS intensity, normalized to unity at the center wave number of the SRL hyperspectral scan range.

To represent the measured SRL spectra as $\mathfrak{\Im}(\overline{\widetilde{\chi}})$, we then used the aqueous buffer PBS as a reference value since the $\mathfrak{\Im}(\overline{\widetilde{\chi}})$ of PBS is known (almost identical to that of water). ${ }^{29}$ For this purpose, the SRL signal at the center wave number, in the region outside the lipid bilayer, was assigned to half the $\mathfrak{I}(\overline{\widetilde{\chi}})$ value of PBS since only one half of the PSF contains the buffer, while the other half contains glass with a negligible $\mathfrak{I}(\overline{\widetilde{\chi}})$. This determined the factor to convert SRL spectra from measured units (volts) into $\mathfrak{\Im}(\overline{\widetilde{\chi}})$ values, as discussed in more detail in the Supporting Information (Section S4.ii).

Figure 5a shows the SRL spectra for the $\mathrm{L}_{\mathrm{o}}$ and $\mathrm{L}_{\mathrm{d}}$ domains obtained on five different regions of the ternary 2:2:1 sample, where nominally identical single lipid bilayers were found. SRL spectra were also measured on control samples consisting of pure DOPC (a thick layer formed by spin-coating) and a SMe homogeneous single bilayer (DOPC/SM/Chol molar ratio $0: 7: 3$ ) and are shown as red curves in Figure 5a (the SMe spectrum is the mean over four nominally identical bilayers). For comparison to the amplitude of the measured CARS spectra discussed in the previous sections, the spectral integral of each SRL spectrum was set equal to that of the corresponding $\mathfrak{I}(\overline{\widetilde{\chi}})$ mean spectrum of the homogeneous bilayer measured by CARS (see Figure 3). We see that SRL spectra for $L_{o}$ and $L_{d}$ coincide with the reference spectra of the homogeneous samples within errors. They are also consistent with the measured CARS spectra, shown as green curves in Figure 5a (taken from the unlabelled ternary mixtures in Figure 4). The observed differences between CARS and SRL spectra are attributed to the different spectral resolutions and pulse temporal/spectral widths in the two measurements. The CARS setup used offered a narrower spectral resolution and a broader wave number range that can be accessed with a good signal-tonoise ratio within the time overlap of the chirped pump and Stokes pulses (see Materials and Methods). As a result, SRS spectra exhibit a less-sharply-rising edge around $2850 \mathrm{~cm}^{-1}$ and have more noise in the spectral region above $2950 \mathrm{~cm}^{-1}$. Otherwise, spectra measured in CARS and SRL have a comparable line shape, confirming the ability of our label-free hyperspectral imaging and unsupervised analysis to consistently differentiate between $\mathrm{L}_{\mathrm{o}}$ and $\mathrm{L}_{\mathrm{d}}$ domains. An example of the spatial maps of the $L_{o}$ and $L_{d}$ components obtained with SRL for one of the five investigated layers is shown in Figure 5 alongside the corresponding DIC differential and integrated optical phase image. Also here, we see excellent correlation between the spatial pattern of thicker domains observed in DIC and the $\mathrm{L}_{\mathrm{o}}$ domains found in SRL. More results are shown in the Supporting Information (Section S5).

\section{CONCLUSIONS}

In summary, we have shown that hyperspectral CRS microscopy, combined with a quantitative unsupervised factorization of the measured data sets, can be used to resolve lipid partitioning and phase-separated domains in single lipid bilayers, both spatially and spectrally. Notably, to date, Raman microscopy of lipid domains in single membranes has largely exploited "Raman tags" to increase chemical specificity and has not yet taken full advantage of the inherently label-free capabilities of the technique.

By applying this analysis to lipid bilayers formed with ternary mixtures of DOPC, SM, and Chol, we have extracted the Raman spectra of $\mathrm{L}_{\mathrm{o}}$ and $\mathrm{L}_{\mathrm{d}}$ domains in susceptibility units relative to a nonresonant medium (glass or PBS) and correspondingly quantified the spatial distribution of the concentration of these components. We find that the spectra of the $\mathrm{L}_{\mathrm{d}}$ components are equal within error to those of pure DOPC. Similarly, the spectra of the $\mathrm{L}_{\mathrm{o}}$ domains are identical to those measured in homogeneous mixtures enriched in SM and Chol. A comparative study between bilayers formed using fluorescently labelled NBD-DOPE versus unlabelled DOPE in the ternary mixture suggests that the fluorescent lipid analogue is affecting the spatial distribution of the domains but not their spectra. It should be noted that the unsupervised factorization does not resolve the individual distribution of SM and Chol within the $\mathrm{L}_{\mathrm{o}}$ domain. To that end, the use of Raman tags on a specific lipid species (e.g., SM) provides useful complementary information.

It is important to point out that imaging planar single bilayers pushes the detection sensitivity of CRS to its limit (as exemplified in Figure 1, comparing the signal from a bulk lipid to that of a single bilayer). This is because the coherent signal enhancement of the technique relies on having a large number of identical chemical bonds in the focal volume, and only a bidimensional layer of chemical bonds is available in a single bilayer. It is therefore remarkable that, despite the low signal levels, hyperspectral CRS imaging does encode sufficient information to retrieve the spectra and concentration of the domains via an unsupervised factorization algorithm with no prior knowledge. 
In the future, this methodology could be applied to a variety of biomimetic membranes, including model systems closer to the complexity of cellular membranes (e.g., incorporating proteins). Notably, CRS is amenable to correlative fluorescence microscopy of tagged proteins in the same instrument, opening the prospect to non-invasively gain new insights into the relationship between lipid domains, their spatially resolved chemical composition, and lipid-protein interactions.

\section{ASSOCIATED CONTENT}

\section{S1 Supporting Information}

The Supporting Information is available free of charge at https://pubs.acs.org/doi/10.1021/acs.analchem.0c03179.

Quantitative DIC acquisition and analysis, CARS and SRS setups, and hyperspectral CARS and SRS analyses (PDF)

\section{AUTHOR INFORMATION}

\section{Corresponding Author}

Paola Borri - School of Biosciences, Cardiff University, Cardiff CF10 3AX, U.K.; 이이.orido000-0002-7873-3314; Email: borrip@cardiff.ac.uk

\section{Authors \\ Alexander Nahmad-Rohen - School of Physics \& Astronomy, Cardiff University, Cardiff CF24 3AA, U.K. \\ David Regan - School of Physics \& Astronomy, Cardiff University, Cardiff CF24 3AA, U.K. \\ Francesco Masia - School of Biosciences, Cardiff University, Cardiff CF10 3AX, U.K. \\ Craig McPhee - School of Biosciences, Cardiff University, Cardiff CF10 3AX, U.K. \\ Iestyn Pope - School of Biosciences, Cardiff University, Cardiff CF10 3AX, U.K. \\ Wolfgang Langbein - School of Physics \& Astronomy, Cardiff University, Cardiff CF24 3AA, U.K.; 10 orcid.org/0000-0001- 9786-1023}

Complete contact information is available at:

https://pubs.acs.org/10.1021/acs.analchem.0c03179

\section{Notes}

The authors declare no competing financial interest. Information on the data presented here, including how to access them, can be found in the Cardiff University data archive http://doi.org/10.17035/d.2020.0112949840.

\section{ACKNOWLEDGMENTS}

The microscope setup used here was developed with funding from the UK BBSRC (grant no. BB/H006575/1) and EPSRC (grant no. EP/M028313/1). D.R. acknowledges funding by an EPSRC DTA studentship (grant no. EP/M507842/1). P.B. and C.M. acknowledge the EPSRC for P.B.'s Leadership fellowship award (grant no. EP/I005072/1) which funded C.M.'s PhD studentship. W.L. acknowledges support by his Leverhulme Royal Society Research Fellowship (grant no. LT20085). P.B. acknowledges the Royal Society for her Wolfson Research Merit Award (grant no. WM140077). F.M. acknowledges the Ser Cymru II programme (Case ID 80762CU-148) which is partly funded by Cardiff University and the European Regional Development Fund through the Welsh Government.

\section{REFERENCES}

(1) Siontorou, C.; Nikoleli, G.-P.; Nikolelis, D.; Karapetis, S. Membranes 2017, 7, 38.

(2) Sezgin, E.; Levental, I.; Mayor, S.; Eggeling, C. Nat. Rev. Mol. Cell Biol. 2017, 18, 361-374.

(3) Rideau, E.; Dimova, R.; Schwille, P.; Wurm, F. R.; Landfester, K. Chem. Soc. Rev. 2018, 47, 8572-8610.

(4) van Meer, G.; Voelker, D. R.; Feigenson, G. W. Nat. Rev. Mol. Cell Biol. 2008, 9, 112-124.

(5) Alessandrini, A.; Facci, P. Soft Matter 2014, 10, 7145-7164.

(6) Mashaghi, A.; Mashaghi, S.; Reviakine, I.; Heeren, R. M. A.; Sandoghdar, V.; Bonn, M. Chem. Soc. Rev. 2014, 43, 887-900.

(7) Regan, D.; Williams, J.; Borri, P.; Langbein, W. Langmuir 2019, $35,13805-13814$.

(8) McPhee, C. I.; Zoriniants, G.; Langbein, W.; Borri, P. Biophys. J. 2013, 105, 1414-1420.

(9) Sezgin, E.; Levental, I.; Grzybek, M.; Schwarzmann, G.; Mueller, V.; Honigmann, A.; Belov, V. N.; Eggeling, C.; Coskun, Ü.; Simons, K.; Schwille, P. Biochim. Biophys. Acta 2012, 1818, 1777-1784.

(10) Faller, R. Biochim. Biophys. Acta, Biomembr. 2016, 1858, 23532361.

(11) Ayuyan, A. G.; Cohen, F. S. Biophys. J. 2006, 91, 2172-2183.

(12) Opilik, L.; Bauer, T.; Schmid, T.; Stadler, J.; Zenobi, R. Phys. Chem. Chem. Phys. 2011, 13, 9978-9981.

(13) Pahlow, S.; März, A.; Seise, B.; Hartmann, K.; Freitag, I.; Kämmer, E.; Böhme, R.; Deckert, V.; Weber, K.; Cialla, D.; Popp, J. Eng. Life Sci. 2012, 12, 131-143.

(14) Ando, J.; Kinoshita, M.; Cui, J.; Yamakoshi, H.; Fujita, K.; Murata, M.; Sodeoka, M. Proc. Natl. Acad. Sci. U.S.A. 2015, 112, $4558-4563$

(15) Donaldson, S. H.; de Aguiar, H. B. J. Phys. Chem. Lett. 2018, 9, $1528-1533$

(16) Zumbusch, A.; Langbein, W.; Borri, P. Prog. Lipid Res. 2013, 52, 615-632.

(17) Camp, C. H.; Cicerone, M. T. Nat. Photon. 2015, 9, 295-305.

(18) Li, L.; Wang, H.; Cheng, J.-X. Biophys. J. 2005, 89, 3480-3490.

(19) Potma, E. O.; Xie, X. S. ChemPhysChem 2005, 6, 77-79.

(20) Masia, F.; Glen, A.; Stephens, P.; Borri, P.; Langbein, W. Anal. Chem. 2013, 85, 10820-10828.

(21) Wurpel, G. W. H.; Schins, J. M.; Müller, M. J. Phys. Chem. B 2004, 108, 3400-3403.

(22) Rinia, H. A.; Bonn, M.; Müller, M.; Vartiainen, E. M. ChemPhysChem 2007, 8, 279-287.

(23) Pope, I.; Langbein, W.; Watson, P.; Borri, P. Opt. Express 2013, 21, 7096-7106.

(24) Langbein, W.; Regan, D.; Pope, I.; Borri, P. APL Photonics 2018, 3, 092402.

(25) Di Napoli, C.; Pope, I.; Masia, F.; Langbein, W.; Watson, P.; Borri, P. Anal. Chem. 2016, 88, 3677-3685.

(26) Karuna, A.; Masia, F.; Wiltshire, M.; Errington, R.; Borri, P.; Langbein, W. Anal. Chem. 2019, 91, 2813-2821.

(27) Rocha-Mendoza, I.; Langbein, W.; Borri, P. Appl. Phys. Lett. 2008, 93, 201103.

(28) Masia, F.; Karuna, A.; Borri, P.; Langbein, W. J. Raman Spectrosc. 2015, 46, 727-734.

(29) Karuna, A.; Masia, F.; Borri, P.; Langbein, W. J. Raman Spectrosc. 2016, 47, 1167-1173.

(30) Pan, J.; Tristram-Nagle, S.; Kučerka, N.; Nagle, J. F. Biophys. J. 2008, 94, 117-124.

(31) Veatch, S. L.; Keller, S. L. Phys. Rev. Lett. 2005, 94, 148101.

(32) Baumgart, T.; Hunt, G.; Farkas, E. R.; Webb, W. W.; Feigenson, G. W. Biochim. Biophys. Acta 2007, 1768, 2182-2194.

(33) Pope, I.; Payne, L.; Zoriniants, G.; Thomas, E.; Williams, O.; Watson, P.; Langbein, W.; Borri, P. Nat. Nanotechnol. 2014, 9, 940946.

(34) Kamble, S.; Patil, S.; Kulkarni, M.; Murthy, A. V. R. Colloids Surf., B 2019, 176, 55-61.

(35) Marquês, J. T.; Viana, A. S.; De Almeida, R. F. M. Biochim. Biophys. Acta 2011, 1808, 405-414. 
(36) Li, L.; Cheng, J.-X. J. Phys. Chem. B 2008, 112, 1576-1579.

(37) Di Napoli, C.; Masia, F.; Pope, I.; Otto, C.; Langbein, W.; Borri, P. J. Biophot. 2014, 7, 68-76.

(38) Collard, L.; Sinjab, F.; Notingher, I. Biophys. J. 2019, 117, $1589-1598$

(39) Czamara, K.; Majzner, K.; Pacia, M. Z.; Kochan, K.; Kaczor, A.; Baranska, M. J. Raman Spectrosc. 2014, 46, 4-20.

(40) Quinn, P. J.; Wolf, C. J. Phys. Chem. B 2010, 114, 1553615545 . 